\title{
La Revista de Neuro-Psiquiatría y los primeros tratamientos biológicos en la psiquiatría mundial.
}

\author{
The Revista de Neuro-Psiquiatría and the first biological treatments in world psychiatry. \\ Santiago Stucchi-Portocarrero ${ }^{1, a}$ \\ RESUMEN
}

A lo largo de las primeras décadas de su existencia, la Revista de Neuro-Psiquiatría fue testigo y fuente efectiva de difusión de los trascendentales avances en la terapéutica psiquiátrica que se dieron entre los años de 1930 a 1960, particularmente en el campo de los tratamientos biológicos. Estos avances condujeron a que la psiquiatría, aun cuando no sin tropiezos u obstáculos, fuera considerada, con toda justicia, como una especialidad médica.

PALABRAS CLAVE: Psiquiatría, tratamiento, historia.

\section{SUMMARY}

During the first decades of its existence, the Revista de Neuro-Psiquiatria became both a witness and an effective source of dissemination of the significant advances in psychiatric treatment that occurred between the 1930s and 1960s, particularly in the area of biological treatments. These advances led psychiatry, even though not without obstacles or setbacks, to being considered, in all fairness, as a medical specialty.

KEYWORDS: Psychiatry, treatment, history.

\section{INTRODUCCIÓN}

“Qué es la historia?” se pregunta Edward Carr en su obra que lleva justamente aquel título (1). Tradicionalmente, se le ha considerado como la captación y registro, por parte de un imparcial compilador (el historiador), de hechos descritos en documentos incontrovertibles calificados apropiadamente como "históricos". Tal definición peca sin embargo de cierta inexactitud. ¿Cómo se definen por ejemplo -según Carr- los criterios que deslinden entre los hechos históricos y los hechos comunes? Es más, en la priorización misma de los acontecimientos ya definidos como históricos, la influencia de la ideología del historiador es un hecho ineludible. Como ineludible resulta también el sesgo que el constructor de la historia imprimirá en la interpretación de aquellos hechos, elemento esencial que diferencia entre la historia y la simple cronología y, todo aquello, asumiendo que las fuentes son irrefutablemente verídicas. Sin embargo -y para complicar todavía más el panorama-, ¿no fueron aquellas fuentes asimismo escritas y seleccionadas previamente por humanos tan proclives a la subjetividad como nosotros? Carr concluye entonces que la historia es "un proceso continuo de interacción entre el historiador y sus hechos, un diálogo sin fin entre el presente y el pasado".

\footnotetext{
1. Instituto Nacional de Salud Mental "Honorio Delgado - Hideyo Noguchi". Universidad Peruana Cayetano Heredia. Lima, Perú.

a. Médico psiquiatra.
} 
Permítasenos entonces -pese a tan aparentemente desalentador primer párrafo- intentar de todos modos una reconstrucción parcial de las primeras décadas de la Revista de Neuro-Psiquiatría, en lo referente a la publicación de artículos científicos sobre métodos terapéuticos auténticamente innovadores dentro de la psiquiatría, en el marco del arrollador avance de los tratamientos biológicos durante el segundo tercio del siglo XX. Con esta delineación más o menos precisa, me adelanto (creo) a las posibles críticas de subjetividad en torno a la selección de los artículos, así como a la interpretación sobre la trascendencia de los mismos.

Ya desde el título caemos en controversia. ¿Cómo definimos los tratamientos biológicos sin contraponerlos a la idea de tratamientos psicológicos, aludiendo así al ahora impopular concepto de dualismo cuerpo/mente, de lejanas pero persistentes reminiscencias cartesianas? Señalo aquí, de la manera más clara posible, que mente y soma no son más que aspectos de un mismo fenómeno, no siendo entonces concebible la existencia de una psiquis "flotante" o carente de una arquitectura orgánica que la sostenga. Coincidimos de este modo con autores como Kendler, el cual rechaza aquella dicotomía en su renovada versión "cerebro-hardware / mentesoftware" y propone una interesante alternativa (2) cuya descripción y discusión rebasan los alcances de este artículo.

En todo caso, el concepto dualista no ha sido del todo invalidado, por lo cual utilizaremos aquí la frase "tratamientos biológicos" para referirnos a todas aquellas modalidades terapéuticas (o que pretenden ser terapéuticas) que actúan directamente en el organismo, mediante agentes físicos, químicos o bioquímicos, con el propósito de modificar la conducta u otra función mental considerada como patológica. En tal sentido, el siglo XX fue pródigo en tales procedimientos, desde la aparentemente tenebrosa malarioterapia hasta los primeros psicofármacos, pasando por el coma insulínico, los choques inducidos por drogas convulsivantes y electricidad, así como la vilipendiada psicocirugía. No todos fueron laureles por supuesto, y quizás haya mucho de lo cual avergonzarse, pero una vez más, eso ya es parte del diálogo entre el pasado y el presente.

\section{Las convulsiones químicas}

Honorio Delgado inauguró la Revista de NeuroPsiquiatría en 1938, con un reporte referente al uso del agente convulsivante pentametilentetrazol (Cardiazol) como tratamiento supuestamente curativo de la esquizofrenia, método que había sido introducido por el húngaro Ladislaus von Meduna en 1937, bajo la premisa (luego desestimada) de un supuesto antagonismo entre la epilepsia y la psicosis (3). Veinticinco individuos con diagnóstico de esquizofrenia fueron sometidos a varias aplicaciones de tal medicamento, completándose el tratamiento en 22 de ellos. Once de estos 22 padecían la enfermedad por menos de un año y medio, obteniendo todos una remisión del cuadro, en tanto que de los 11 restantes, con una duración de enfermedad entre año y medio y 8 años, sólo remitieron 5 .

En otro artículo del mismo año, Anglas Quintana describe minuciosamente el procedimiento en cuestión tras haberlo aplicado aproximadamente 30 veces en cada uno de 32 pacientes esquizofrénicos (4). Concluye el investigador que la "Cardiazolterapia en la esquizofrenia -aunque aparatosa e impresionantees de aplicación sencilla y no entraña peligro alguno en pacientes cuidadosamente seleccionados". Tiempo después, el porcentaje de fracturas generadas por tal método haría dudar de tal seguridad (5). Anglas reconoce, no obstante, que los ataques convulsivos inducidos químicamente "parecen ser más enérgicos y más potentes".

También en 1938 Carlos Krumdieck publicó sus experiencias con el método de Von Meduna en 35 pacientes esquizofrénicas, describiendo "curación" en 17 de ellas, alivio en 7 y falta de respuesta en 11, correspondiendo la mayor parte del éxito a aquellas con menos de 1 año de enfermedad (6). Krumdieck fue sin embargo más allá de la esquizofrenia, reportando buena respuesta con el pentametilentetrazol en dos casos de psicosis puerperal, en una paciente "psicopática antisocial con rasgos esquizofrenoides", en dos "psicasténicas", en una diagnosticada como "histérica de tipo hístero-esquizofrénica" y en otra con manía aguda, mas no en una paciente "psicopática polimorfamente perversa en su aspecto psicosexual". Igualmente innovador fue Federico Sal y Rosas al utilizar el método de Von Meduna en pacientes con histeria y epilepsia; en esta última patología, el autor aseguró haber "reducido la cifra global de ataques a menos de la décima parte de la cantidad de éstos anterior al experimento" $(7,8)$.

Krumdieck finaliza su artículo con frases pletóricas de optimismo en relación a la convulso-terapia química: "Von Meduna ha abierto una vía regia en el 
tratamiento de la alienación mental, particularmente de la esquizofrenia, siendo de esperar que nuevas conquistas en el campo terapéutico, inspiradas en el descubrimiento del sabio húngaro, permitirán dominar la más terrible de las enfermedades conocidas..." (6). Ello no obstante, esta modalidad de tratamiento biológico se eclipsó relativamente pronto con la aparición y difusión del electrochoque, a partir de 1938.

\section{La insulinoterapia}

Fue también en 1938 que Honorio Delgado, Juan Francisco Valega y Carlos Gutiérrez Noriega publicaron en la Revista, sus hallazgos acerca del tratamiento de la esquizofrenia con insulina, método introducido por el psiquiatra polaco Manfred Sakel en 1933; el tratamiento, consistía en aplicar la hormona hasta llevar al paciente al coma hipoglucémico, del cual era luego rescatado mediante infusiones glucosadas (9). De 73 personas con el diagnóstico de esquizofrenia sometidas a insulino-terapia, 45 obtuvieron remisión completa, 9 remisión social, 13 simplemente mejoría y en 6 no hubo modificación; al igual que con las convulsiones químicas, el mayor éxito correspondió a aquellos pacientes con menos tiempo de enfermedad. Los autores reconocen el alto riesgo del coma prolongado ( $21 \%$ de los casos) "con consecuencias casi catastróficas en 6", lamentando inclusive 5 fallecimientos.

A propósito de las complicaciones del método de Sakel, León Mejía dedicó un artículo al tema, encontrando aquéllas en el $80 \%$ de 96 pacientes con esquizofrenia, resaltando las siguientes: "Coma prolongado $(21 \%)$, trastornos respiratorios y circulatorios (14\%), espasmo de la glotis $(6,25 \%)$ ", llegando a un 5,2\% de mortalidad (10). L. A. Guerra por su parte, formuló una serie de descripciones psicopatológicas en 10 individuos sometidos a insulinoterapia, resumiéndolas en: "Manifestaciones de la conciencia (incluyendo la espacial y la temporal); manifestaciones de la afectividad, con predilección por los estados afectivos vitales y por la tendencia afectiva; manifestaciones de la conciencia del yo con predilección por la unidad y por último, de la percepción, de preferencia en el aparato vestibular" (11). Posteriormente, Yépez estudió la respuesta a la insulinoterapia en 118 casos de esquizofrenia clasificados según la tipología somática propuesta por Kretschmer, encontrando un porcentaje mayor de curación entre los pícnicos y normotípicos que entre los leptosómicos (12).
Los estudios nacionales contaron en su momento con el respaldo de la literatura internacional. Por ejemplo, un estudio llevado a cabo en los Estados Unidos en 1939 comparó 1757 pacientes a quienes se les aplicó insulinoterapia con 1140 en quienes se utilizó el método de las convulsiones químicas, describiendo una significativa ventaja para el primer grupo (13). Sin embargo, estudios posteriores hechos a largo plazo encontraron que la supuesta cura era temporal, y con el paso de las décadas, la aparición de los antipsicóticos hizo que el método de Sakel fuera abandonado (5).

\section{El electrochoque y la electronarcosis}

Aunque propuestas de uso médico de la electricidad datan por lo menos del siglo XVIII, la primera inducción intencional de convulsiones generalizadas mediante un choque eléctrico con propósitos terapéuticos fue efectuada por los italianos Ugo Cerletti y Lucio Bini en 1938. En 1943, César Delgado publicó en la Revista una extensa revisión sobre el método, incluyendo su propia experiencia con 10 pacientes (14). Utilizó para ello "un aparato igual al patentado por Cerletti y Bini", con un tiempo de "0.3 de segundo, (...) un voltaje que fluctuaba entre 70 y 90 voltios, y una intensidad entre 350 y 550 miliamperios". En cuanto a los resultados, el autor afirma que "sobrepasan las mejores expectativas", mostrándose el método "particularmente eficaz: a) en la fase melancólica de la psicosis maníaco-depresiva y en la melancolía de involución; b) en la fase maníaca de la psicosis maníaco-depresiva; c) en la histeria; y d) en la esquizofrenia de corta duración". Considera que el método es muy seguro, con ventaja añadida de su bajo costo y reconociendo únicamente alteraciones reversibles de la memoria como efectos secundarios notables.

No debe confundirse el electrochoque (o terapia electroconvulsiva en su más reciente y eufemística denominación) con la electronarcosis; ambos comparten una aplicación eléctrica en la cabeza con propósitos pretendidamente terapéuticos, el primero mediante una descarga muy breve y relativamente elevada de electricidad, seguida siempre por una convulsión tónico-clónica generalizada, en tanto que la segunda aplica una descarga menos intensa y en forma progresiva, no generando necesariamente una convulsión completa. Presentada en su momento como superior y más segura que el electrochoque, la electronarcosis mereció también algunos artículos en la Revista de Neuro-Psiquiatría, tales como el de 
Ottavio Vergani referente al exitoso uso de tal terapia en 138 mujeres del Instituto Psiquiátrico de MilanoAffori (15). En años subsiguientes, sin embargo, la terapia electroconvulsiva no solo predominó sobre la electronarcosis, sino que a la postre, se constituyó en la única terapia psiquiátrica pre-farmacológica que sobreviviría hasta el siglo XXI.

La adición de un relajante muscular con anestesia general otorgó más seguridad a la terapia electroconvulsiva. En este contexto, Aliaga, Querol y Chicata reportaron en 1957 su experiencia con 18 pacientes a quienes se les aplicó electrochoque previa administración de succinilcolina y un barbitúrico de acción ultrarrápida (16).

\section{La psicocirugía}

Julio Óscar Trelles publicó en 1943 un extenso artículo sobre la leucotomía pre-frontal, método neuroquirúrgico introducido por los portugueses Egas Moniz y Almeida Lima en 1935, modificado y popularizado luego por los norteamericanos Walter Freeman y James Watts, con el propósito de corregir disturbios mentales (17). Expone Trelles las técnicas operatorias conocidas, siendo evidente que aún Freeman no había simplificado la técnica hasta convertirla en la lobotomía transorbitaria (conocida más tarde, ingratamente, como la cirugía del "picahielo"). El autor es meticuloso en su revisión de la literatura, no restándole elogios al método, mas siendo a la vez cauto al advertir que el mismo debía considerarse "como reservado a los casos de mal pronóstico y como la última carta a jugar, es una terapéutica de excepción y se la puede considerar como todavía en el terreno de la experimentación". En 1956, Mariano Querol sería categórico: "El furor neuroquirúrgico en el tratamiento de la esquizofrenia ha pasado. (...) La neurocirugía, impropiamente denominada psicocirugía, no es un método curativo de la esquizofrenia" (18).

No les fue posible a Trelles o a Querol vaticinar los excesos a los que llegaría Freeman durante las décadas de 1940 y 1950: más de 3000 lobotomías llevadas a cabo por él mismo aplicando criterios cada vez más laxos hasta que, en 1967, le fue retirada su licencia tras la muerte de uno de sus operados (19).

\section{La clorpromazina}

En 1950 Paul Charpentier sintetizó en Francia la clorpromazina (4650 R.P.), fármaco destinado a convertirse en el primer antipsicótico, tras la publicación de las investigaciones de Henri Laborit, y luego las de Pierre Deniker y Jean Delay (20). La nueva molécula llegó al Perú, "cupiendo a nuestro país ser el primero en usarlo en el Nuevo Mundo" (21). Julio Óscar Trelles y Alfredo Saavedra publicaron en 1954, en la Revista de Neuro-Psiquiatría, las primeras experiencias con aquel medicamento, bajo la modalidad entonces en boga de la cura de sueño. Así, 20 pacientes fueron sometidos a dosis entre 50 y $125 \mathrm{mg}$ de clorpromazina, tanto por vía intramuscular como por vía oral, añadiéndoseles barbitúricos o hidrato de cloral. Los autores reportaron los siguientes resultados: "1. La cura resultó eficaz en las psicosis sintomáticas, en la manía y en la melancolía ansiosa; 2. Puede servir como medida de urgencia en diversos síndromes de agitación (esquizofrénicos, neuróticos, epilépticos), pero en todos estos casos debe seguirse de inmediato de la medicación ya conocida (insulinoterapia, electrochoque, psicoterapia, medicación anticonvulsiva), insustituible hasta ahora; 3. El método es prácticamente inocuo y ayuda francamente en la labor psicoterápica de todos los casos" (21).

La clorpromazina generó un gran entusiasmo, que se plasmó en una considerable cantidad de publicaciones. Carlos Alberto Seguín y Renato Castro presentaron su experiencia en la Sociedad de NeuroPsiquiatría y Medicina Legal, utilizando dosis de clorpromazina entre 75 y $100 \mathrm{mg} /$ día, obteniendo los mejores resultados "en los cuadros de agitación y en las psiconeurosis, siendo relativos en la esquizofrenia y epilepsia" (22). Federico Sal y Rosas, Raúl Jerí y Sánchez García presentaron asimismo en la Sociedad sus experiencias con el fármaco a dosis de 75-100 mg/día, con resultados "halagadores en las neurosis de angustia (25 casos), en los estados de agitación, psicosis sintomáticas" y "relativos" en la esquizofrenia y epilepsia (23). Cabe aclarar que en ambos trabajos se utilizaron dosis relativamente bajas de clorpromazina y por tiempos reducidos, lo cual explicaría la pobre respuesta registrada entonces en pacientes esquizofrénicos.

En un artículo de 1955, Carrillo Broatch reportó 250 casos de esquizofrenia crónica de difícil manejo, sometidos a terapéutica mixta simultánea ("insulinoterapia, electrochoque, lavado ventricular, leucotomía, Largactil, Serpasol, etc.), en las más variadas combinaciones, lo cual hizo difícil el extraer conclusiones acerca de la efectividad aislada de cada uno de los métodos (24). 
En 1956 Querol, Samanez y Almeida reportaron el uso de clorpromazina (sola o asociada a sedantes o electroplexia) en un grupo de 26 esquizofrénicos y 6 maniaco-depresivos, en dosis de 100 a $600 \mathrm{mg} /$ día y por un tiempo mínimo de 3 meses, todo lo cual establecía una diferencia importante con las experiencias anteriores (25). Los resultados fueron alentadores, llevando a los autores a afirmar "que la clorpromacina deba ser considerada como un tratamiento fundamental en estas psicosis endógenas". En otro artículo, Querol y Chicata encontraron también una significativa ventaja de la clorpromazina al comparar 13 pacientes utilizando dicho medicamento con 46 que recibieron otros tratamientos (17). Luego Chicata publicó su experiencia con 45 esquizofrénicos y 9 maniaco-depresivos a quienes se les administró clorpromazina en dosis de 75 a $800 \mathrm{mg} /$ día, sola o asociada a electrochoque, insulinoterapia o reserpina, encontrando remisión completa en el $80 \%$ de esquizofrénicos con enfermedad menor de un año, y en el 37,5\% de aquellos con más tiempo de enfermedad; por su parte, todos los casos de manía remitieron en un lapso de 1 a 3 meses (26).

Arnaldo Cano, Elsa Felipa y Manuel Escobar utilizaron la clorpromazina en 106 casos de neurosis, en dosis y tiempos muy variables, desde 100 hasta $1200 \mathrm{mg} /$ día, y desde 3 días hasta 18 meses, respectivamente, reportando remisión total en el $28 \%$, remisión parcial en el $26 \%$, falta de remisión en el $36 \%$ y evolución ignorada en el 10\% (27). Concluyen además que la clorpromazina "es eficaz en síndromes reactivos esencialmente exógenos; menos eficaz cuando hay elementos depresivos que cuando sólo hay ansiedad o angustia". Almeida por su parte, empleó el mismo fármaco para el tratamiento de 59 alcohólicos "de los cuales 30 eran intoxicados agudos en estado de excitación, 16 tenían síntomas post-alcohólicos y 13 con cuadros de psicosis alcohólicas", consiguiendo "atenuar los síntomas de inquietud psicomotriz, la angustia, las náuseas y vómitos y el insomnio" (28). Emilio Majluf describió el uso de la clorpromazina en niños con "psicosis, deficiencia mental, trastornos de conducta primarios y secundarios, tartamudez, epilepsia, parálisis cerebrales, etc.", a dosis de 100$200 \mathrm{mg}$ /día, "con resultados en general satisfactorios" (29).

No pasó mucho tiempo para que empezaran a manifestarse los efectos secundarios de la hasta entonces supuestamente inocua clorpromazina. De este modo, Trelles y Víctor Saavedra presentaron en 1957 ante la Sociedad de Neuro-Psiquiatría y Medicina Legal, el caso de una paciente con síndrome parkinsoniano y crisis oculocefalogíricas inducidas por dicho fármaco. El comentario de Raúl Jerí es revelador: "Las drogas ataráxicas cada día presentan más complicaciones tóxicas. Hace dos años pensábamos que la clorpromazina no producía alteraciones" (30).

\section{La reserpina}

Aunque desde tiempos inmemoriales se utilizaba medicinalmente en la India la planta denominada Pagal-Ka-Dawa ("hierba para la locura"), fue en 1954 que el alcaloide activo denominado reserpina ingresó a la clínica psiquiátrica como agente antipsicótico (31). Su uso en nuestro país fue prontamente reportado por Jerí, en 1955, tras haber empleado la reserpina en 21 pacientes psiquiátricos y 4 voluntarios sanos, aplicada en forma endovenosa y oral según el estado clínico, y en dosis de mantenimiento de 4-8 mg/día; en "todos los casos donde existía agitación, excitación o excesiva tensión psíquica...(se demostró)...rápida remisión de los síntomas, unas veces en forma muy considerable, en otras oportunidades el efecto ha sido moderado" (32). El mismo Jerí reportó en 1957 el uso de reserpina en 159 casos de psicosis, con dosis iniciales (por vía oral o intramuscular) de $10-30 \mathrm{mg} /$ día y de mantenimiento de 1-8 $\mathrm{mg} /$ día, por un período de hasta 3 años, describiendo una acción fundamental "sobre estados de ansiedad, tensión, inquietud, excitación y agitación psicomotriz. Los efectos no son constantes y los desórdenes psicopatológicos primarios no se modifican" (33). En una breve comunicación, Majluf expuso su experiencia con la reserpina a dosis de 5-10 mg/día en niños "que exhibían inquietud, agresividad $\mathrm{y}$ tendencias destructivas, asimismo en algunos psicóticos", obteniendo "por lo general buenos resultados, pero es nuestra impresión que son inferiores a los obtenidos con la clorpromacina" (29).

El paso de la reserpina por el sendero de la psiquiatría fue sin embargo efímero, cayendo en desuso luego de atribuírsele algunos casos de depresión y suicidio.

\section{La imipramina}

Honorio Delgado mencionó en 1960 el uso de la imipramina en el tratamiento de la melancolía, a una dosis mínima de 50-200 mg/día y máxima de 75$400 \mathrm{mg} / \mathrm{día}$, notando que el "síntoma que desaparece primero es la angustia, con más frecuencia que en los enfermos tratados con electrochoque. Asimismo, la propensión al suicidio cede muy pronto, pudiendo 
recrudecer si se interrumpe la administración del fármaco" (34). Este medicamento había sido introducido como antidepresivo en 1958 por el investigador suizo Roland Kuhn, pionero de este grupo de psicofármacos. En el mismo número de la Revista de Neuro-Psiquiatría, Alfredo Saavedra, Javier Mariátegui y Luis Boggiano publicaron su experiencia en 40 pacientes "de los cuales 30 han sido catalogados dentro del círculo de la psicosis maniacodepresiva, 9 dentro de la melancolía de involución y 1 como perteneciente a la depresión orgánica" (34); se administró imipramina a una dosis media de 200 $\mathrm{mg}$ /día y máxima de $400 \mathrm{mg} /$ día, iniciando con la vía intramuscular y continuando oralmente, por un tiempo de hasta 18 semanas, logrando finalmente buena respuesta terapéutica en 25 pacientes y apreciándose "mayor eficacia de la droga en los casos de melancolía de involución y en los casos de P.M.D. que sólo evolucionan con episodios depresivos" (35).

Delgado comparó luego el electrochoque con la imipramina, hallando "remisión completa o mejoría notable" con el primero en el $66,1 \%$, y con la segunda en el $56 \%$ de los 56 casos de melancolía estudiados (36). Fernando Osorio reportó 9 casos de depresión tratados con el nuevo antidepresivo, todos con muy buena respuesta (37). Por su parte, Trelles, S. Escalante y Edmundo Beteta utilizaron la imipramina para el tratamiento de la depresión reactiva en personas con diferentes trastornos neurológicos (síndromes piramidales, extrapiramidales y cerebelosos), señalando "algunas respuestas terapéuticas favorables principalmente en los cuadros parkinsonianos y piramidales", aunque con no pocos efectos colaterales, incluyendo convulsiones generalizadas, incremento de los movimientos involuntarios e hipotensión arterial (38).

\section{La carbamazepina}

En 1967 Javier Mariátegui, Hugo Chávez y José Oballe reportaron el uso de la carbamazepina -fármaco cuyas propiedades antiepilépticas y antineurálgicas habían sido descritas desde 1963- en 32 pacientes, "24 con diversas formas paroxismales de epilepsia y 8 con alteraciones del comportamiento de base comicial", resaltando la necesidad de un medicamento "con propiedades a la vez anticonvulsivas y psicotrópicas" (39). Con una dosis media de $600 \mathrm{mg} /$ día y un tiempo de tratamiento promedio de 6,5 meses, en "las formas paroxismales los resultados fueron excelentes en el $58,4 \%$ y buenos en el 33,3\%; en las alteraciones de conducta, excelentes en el 12,5\% y buenos en el 50\%".
Roedenbeck y Santos utilizaron la carbamazepina en 15 niños de 2 a 11 años de edad con crisis epilépticas rebeldes a otros tratamientos; aunque los resultados fueron "difíciles de analizar, comparar y evaluar, debido al corto tiempo de observación y a que los casos tratados son heterogéneos", de todos modos se reportó desaparición de las crisis en 6 niños y mejoría en el mismo número (40). En otro estudio, Sánchez, Vásquez y Aréstegui administraron la carbamazepina (dosis de 600 hasta $1200 \mathrm{mg} /$ día por un periodo de 5 meses) a 18 epilépticos crónicos con mala respuesta a tratamientos previos y, además, "inadecuación en las relaciones interpersonales, retardo psicomotor, distimias, agresividad en sus diversas formas, viscosidad; unos pocos habían tenido, en algún momento, intentos suicidas u homicidas" (41). Los resultados demostraron control de las crisis epilépticas en 7 de 13 casos con crisis presentes al iniciarse el estudio; en cuanto a modificaciones del cuadro psicopatológico, se obtuvo respuesta muy buena en 8 casos, buena en 4 , regular en 5 y nula en 1 , describiendo los autores "una disminución de la agresividad, las distimias desaparecieron o disminuyeron en intensidad y frecuencia; se observó, asimismo, mejoría en las relaciones interpersonales".

\section{Otros psicofármacos}

$\mathrm{Al}$ referirnos a los psicofármacos, hemos centrado la presente revisión en aquellos considerados como pioneros en su grupo terapéutico. Nos resistimos sin embargo a dejar de lado los reportes referidos a otros medicamentos de particular relevancia en la farmacopea psiquiátrica, tales como el meprobamato, mencionado por Baltazar Caravedo Carranza (42); la tioridazina, estudiada en las publicaciones de Alfredo Saavedra y Javier Mariátegui (43) y Emilio Majluf (44); la amitriptilina, objeto de estudio por parte del mismo Mariátegui y E. Ramírez del Villar (45), y Segisfredo Luza (46); el haloperidol, utilizado por Grover Mori (47) y Raúl Jerí (48); la flufenazina de acción prolongada, estudiada también por Mariátegui, junto con Renato Alarcón, José Arana, Carlos Azcarate y Alberto Butler (49), y finalmente el tiotixeno, citado en las publicaciones de Mariano Querol, Maíta García, Renato Alarcón, Ángel Infante y Lucía Reyna (50), Ernst Raab y Javier Mariátegui (51) y Alberto Butler (52). Quedan indudablemente sin mencionar otros valiosos trabajos.

\section{CONCLUSIONES}

Es claro que las primeras décadas de la existencia de la Revista de Neuro-Psiquiatría estuvieron 
imbuidas de un optimismo desbordante por los nuevos métodos terapéuticos del siglo XX, particularmente las convulsiones químicas, la insulinoterapia, el electrochoque y los primeros psicofármacos. En tal contexto, la más antigua revista médica peruana cumplió con el objetivo fundamental de mantenerse a la vanguardia del conocimiento en todos los aspectos de su cobertura académica y científica.

Importados de la lejana Europa para su pronta aplicación en el Perú, gracias a la diligencia de los fundadores de la Revista y de sus discípulos y colaboradores, estos métodos de tratamiento encontraron en esta publicación peruana su medio de difusión por antonomasia. Tal optimismo (que hizo decir a Julio Óscar Trelles: "Sin caer en hipérbole se puede afirmar que, en la actualidad, la medicina mental dispone de más armas terapéuticas y obtiene mejores resultados que cualquier otra de las especialidades") (17), se vio contrastado luego por el progresivo abandono de casi todos los métodos pre-farmacológicos (excepto el electrochoque en su actual versión asociada al uso de agentes relajantes musculares y anestésicos) y por la detección de múltiples efectos secundarios de los medicamentos psiquiátricos.

Mezquino sería no obstante, restar méritos a tal empeño; aunque, como se mencionó en la Introducción, resulta ineludible la crítica con lentes contemporáneos (los cuales nos harían observar por ejemplo la metodología no enmarcada en los rígidos parámetros de los actuales ensayos aleatorizados y doble ciego, o preguntarnos tal vez sobre el grado de conocimiento y consentimiento que tuvieron los participantes de los estudios presentados), no puede negarse el valor que las investigaciones sobre los métodos primigenios tuvieron en su momento y la influencia que pudieron haber legado a la posterior aparición de otras terapias actualmente vigentes. Tan importante como todo ello, es el hecho de que los tratamientos biológicos contribuyeron en buena medida a una mayor (aunque no total) aceptación de la psiquiatría como respetable especialidad médica. El presente pues se nutre del pasado, y mal haríamos en renegar de aquél en forma absoluta.

Correspondencia: stucchi@amauta.rcp.net.pe.

\section{REFERENCIAS BIBLIOGRÁFICAS}

1. Carr EH. ¿Qué es la historia? México: Editorial Artemisa SA de CV; 1985.

2. Kendler KS. The dappled nature of causes of psychiatric illness: replacing the organic-functional/ hardware-software dichotomy with empirically based pluralism. Molecular Psychiatry. 2012; 17: 377-88.

3. Delgado H. Tratamiento de la esquizofrenia con Cardiazol a dosis convulsivante. Rev Neuropsiquiatr. 1938; 1(1): 19-50.

4. Anglas P. Aspecto neurológico del ataque provocado por el Cardiazol en esquizofrénicos. Rev Neuropsiquiatr. 1938; 1 (3): 420-44.

5. Sabbatini RME. The history of shock therapy in psychiatry. Brain \& Mind.1997; 4. URL disponible en: http://www.cerebromente.org.br/n04/historia/ shock_i.htm (Fecha de acceso: octubre de 2013).

6. Krumdieck CF. Sobre el tratamiento de la alienación mental por el Cardiazol a dosis convulsivante. Rev Neuropsiquiatr. 1938; 1 (4): 504-15.

7. Sal y Rosas F. Tratamiento de las neurosis por el Cardiazol. Rev Neuropsiquiatr. 1938; I (4): 533-50.

8. Sal y Rosas F. El tratamiento de la epilepsia por el Cardiazol. Rev Neuropsiquiatr. 1939; 2 (1): 81-96.

9. Delgado H, Valega JF, Gutiérrez-Noriega C. Contribución al tratamiento de la esquizofrenia con insulina. Rev Neuropsiquiatr 1938; 1 (4): 463-98.

10. Mejía L. Complicaciones del tratamiento de la esquizofrenia por el método de Sakel. Rev Neuropsiquiatr. 1939; 2 (4): 552-72.

11. Guerra LA. Observaciones psicopatológicas en pacientes tratados con insulina. Rev Neuropsiquiatr. 1942; 5 (2): 75-114.

12. Yépez HM. Influencia de la constitución somática sobre el resultado inmediato de la insulinoterapia de los esquizofrénicos. Rev Neuropsiquiatr. 1944; 7(3): 310-29.

13. Ross JR, Malzberg B. A review of the results of the pharmacological shock therapy and the Metrazol convulsive therapy in New York State. Am J Psychiatry. 1939; 96: 297-316.URL disponible en : http://ajp.psychiatryonline.org/article.aspx?article ID=141761 (Fecha de acceso: octubre del 2013).

14. Delgado C. Tratamiento de las enfermedades mentales por electro-shock. Rev Neuropsiquiatr. 1943; 6 (3): 263-316.

15. Vergani O. La electronarcosis. Rev Neuropsiquiatr. 1951; 14 (3): 349-78.

16. Aliaga P, Querol M, Chicata M. Succinilcolina en los tratamientos de choque. Rev Neuropsiquiatr. 1957; 20(4): 440-50.

17. Trelles JO. La leucotomía pre-frontal de Egaz Moniz. Rev Neuropsiquiatr. 1943; 6(3): 328-63.

18. Querol M. Consideraciones acerca de algunos de los métodos actuales del tratamiento de la esquizofrenia. Rev Neuropsiquiatr .1956; 19(3): 344-61.

19. Gajilan AC. Survivor recounts lobotomy at age 12 . Procedure once considered legitimate medical treatment. CNN. November 30, 2005. URL disponible en: http://www.cnn.com/2005/HEALTH/ 
conditions/11/30/ pdg.lobotomy/index.html. (Fecha de acceso: octubre de 2013).

20. Ban TA. Fifty years of chlorpromazine: a historical perspective. Neuropsychiatr Dis Treat. 2007; 3 (4): 495-500.

21. Trelles JO, Saavedra A. Cura de sueño con Largactil en el tratamiento de algunos desórdenes mentales. (Primeros resultados en el Perú). Rev Neuropsiquiatr. 1954; 17(2): 125-61.

22. Seguín CA, Castro R. Experiencia en el empleo de la Clorpromazina. Rev Neuropsiquiatr. 1954; 17(3): 357.

23. Sal y Rosas F, Jerí R, Sánchez García. Primeros resultados del tratamiento de Largactil en los enfermos mentales. Rev Neuropsiquiatr. 1954; 17(3): 358.

24. Carrillo-Broatch A. Tratamiento de la esquizofrenia crónica. Rev Neuropsiquiatr 1955; 18(4): 441-5.

25. Querol M, Samanez F, Almeida M. Clorpromacina en el tratamiento de la esquizofrenia. Rev Neuropsiquiatr. 1956; 19(4): 518-36.

26. Chicata MA. La clorpromacina en las psicosis endógenas. Rev Neuropsiquiatr. 1957; 20(3): 280-95.

27. Cano A, Felipa E, Escobar M. Clorpromacina en las neurosis. Rev Neuropsiquiatr. 1957; 20(3): 296-305.

28. Almeida M. Empleo de la clorpromacina en el alcoholismo. RevNeuropsiquiatr. 1957; 20(3): 30642.

29. Majluf E. Las nuevas drogas en psiquiatría infantil. Rev Neuropsiquiatr. 1957; 20(4): 421-38.

30. Trelles JO, Saavedra V. Síndrome parkinsoniano con crisis oculocefalogiras en el tratamiento con Clorpromazina. Rev Neuropsiquiatr. 1957; 20(2): 121-7.

31. Álamo C, López Muñoz F, Bhatara VS, Cuenca E. La contribución de la reserpina al desarrollo de la psiquiatría y su papel en la investigación neurobiológica. Rev Psiquiatría Fac Med Barna. 2004; 31(3): 130-9.

32. Jerí R. Observaciones con la reserpina (Serpasol) en sujetos sanos y en pacientes psiquiátricos. Rev Neuropsiquiatr. 1955; 18(1): 108-24.

33. Jerí R. Experiencias con la reserpina en el tratamiento de algunas psicosis endógenas y sintomáticas. Rev Neuropsiquiatr. 1957; 20(3): 342-58.

34. Delgado H. Acerca del tratamiento de las depresiones. Rev Neuropsiquiatr. 1960; 23(2): 157-64.

35. Saavedra A, Mariátegui J, Boggiano L. La imipramina en los estados depresivos. Rev Neuropsiquiatr. 1960; 22(2): 195-228.

36. Delgado H. Tratamiento de la melancolía. Rev Neuropsiquiatr. 1960; 23(3): 291-300.

37. Osorio F. Estados depresivos en pacientes hospitalizados y ambulatorios tratados con Tofranil. Rev Neuropsiquiatr. 1960; 23(3): 363-7.
38. Trelles JO, Escalante S, Beteta E. La imipramina en la práctica neurológica. Rev Neuropsiquiatr. 1960; 23(3): 301-13.

39. Mariátegui J, Chávez H, Oballe J. Acerca de un nuevo anticonvulsivo psicótropo. Resultados preliminares con la carbamazepina. Rev Neuropsiquiatr. 1967; 30(2): 189-97.

40. Roedenbeck SD, Santos J. El Tegretol en el tratamiento de las convulsiones graves infantiles. Rev Neuropsiquiatr. 1967; 30(3): 290-301.

41. Sánchez E, Vásquez G, Aréstegui A. Estudio objetivo de los cambios del comportamiento bajo la acción de la carbamacepina. Rev Neuropsiquiatr. 1967; 30(4): 356-371.

42. Caravedo B. El meprobamato en psiquiatría. Rev Neuropsiquiatr. 1957; 20(3): 393-9.

43. Saavedra A, Mariátegui J. La tioridacina en psiquiatría. Primeros resultados terapéuticos. Rev Neuropsiquiatr. 1959; 22(4): 585-630.

44. Majluf E. La tioridacina en psiquiatría infantil. Rev Neuropsiquiatr. 1961; 24(3): 290-309.

45. Mariátegui J, Ramírez del Villar E. Tratamiento de los cuadros depresivos con amitriptilina. Rev Neuropsiquiatr. 1962; 25(3): 281-90.

46. Luza S. El uso de la amitriptilina en pacientes ambulatorios. Rev Neuropsiquiatr. 1962; 25 (4): 395402.

47. Mori G. El haloperidol en el tratamiento del síndrome maniaco. Rev Neuropsiquiatr. 1966; 29(1): 24-32.

48. Jerí R. El uso de haloperidol en la esquizofrenia residual y en los estados paranoides. Rev Neuropsiquiatr. 1970; 33(2): 112-22.

49. Mariátegui J, Alarcón R, Arana J, Azcarate C, Butler A. Ensayo terapéutico con un derivado fenotiacínico de acción prolongada. El enantato de flufenacina en un grupo de esquizofrénicos crónicos. Rev Neuropsiquiatr .1966; 29(1): 33-44.

50. Querol M, García M, Alarcón R, Infante A, Reyna L. Ensayo terapéutico con una nueva droga antipsicótica (P-4657-B). Rev Neuropsiquiatr. 1968; 31(2): 73-82.

51. Raab E, Mariátegui J. Tiotixeno en el tratamiento ambulatorio de desórdenes emocionales. Rev Neuropsiquiatr. 1968; 31(2): 104-10.

52. Butler A. Ensayo terapéutico con el tiotixeno en desórdenes emocionales. Rev Neuropsiquiatr. 1969; 32(2): 120-34.

Recibido: 20/01/2013

Aceptado: 23/05/2013 